\title{
AN APPROACH TO RESOLVE HETEROGENEITY USING RPC IN CLIENT SERVER SYSTEMS
}

\author{
Sakshi Garg \\ PHD Scholar \\ Amity University, Noida, Uttar Pradesh, India, 201313 \\ Shobhika Sejwal, Jatin Solanki, \\ Department of Information Technology, \\ Amity University, Noida, Uttar Pradesh, India, 201313
}

\begin{abstract}
The Client-Server model basic is to circulate processing and is a response to the defects conveyed by the centralized Client-Server model. The mix of information and administrations in this paper are given by Client Server design where the customers are permitted to be isolated from inborn complexities. As the principle parts in this model, client, server and system can be spoken to in the types of 2 layered and 3 layered Client Server (CS) design. The rising improvement in CS frameworks are figured out as to how to deliver better advances that upgrade the idea of this design. Variables that must be considered in the utilization of these frameworks incorporate information security, preparing of clients, specialized support and expenses. This paper will give data about this model as far as its presentation, engineering, late improvement, issues and answers for them. It mainly concentrates on heterogeneity issue which is essential to conquer a considerable measure of bottlenecks officially introduce in conveyed heterogeneous conditions.
\end{abstract}

Keyword - Client Server architecture, distributed computing, Client Server systems, Client Server 2-tier, 3-tier, Interprocess communication.

\section{INTRODUCTION}

With the headway in innovation, Web is ending up plainly particularly more vital nowadays, in which everything is web utilization every now and then [1]. CS framework has limited services and advancement by isolating sharing data elements both server and client. The client requests while the server supplies the administration. In this condition, the information prepared is server dealt, and the outcomes are given back to the clients, i.e. made to accelerate the rate of execution [2]. As example, a printer in a workstation can be appended to a PC (speaking to the clients) whereas different PCs are the servers sharing from it.

Consequently, this paper shall confer about the ClientServer model in Section II, Section III discusses about the issues and Challenges of client server system. Section IV, gives the Proposed Solution to one of the challenges. Section
$\mathrm{V}$ will list down the conclusions deducted from the study and finally Section VI states the Future Scope of this study.

\section{A CLIENT-SERVER SYSTEM}

Clients are portrayed as procedure or program that solicitates for administration or data, and server are depicted as procedure or an application that gives the administration or data. The administration or data i.e. asked for and given between server and client that can be an asset, for example, information, document, objects, display device or controls [3]. Client and server, both may switch parts when a client turns into a server and other way around. Cases of clientserver framework are the web programs, the managing an account administrations and the email framework [4]. For a case, the keeping money administrations where a web program in the PC utilized by a client to get to the saving money benefits advances a demand to the bank's web server [5]. Inter-process communication among client and servers shown in the fig1.

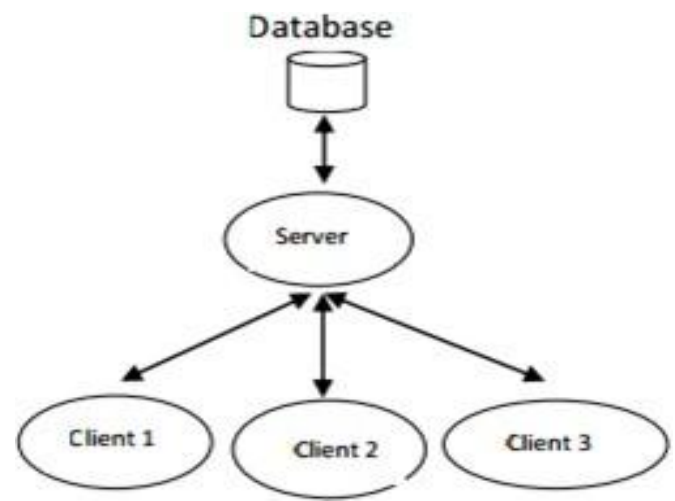

Fig1: Inter-process Communication among Client and Server 


\section{CHALLENGES AND ISSUES IN CLIENT SERVER SYSTEM}

There are a few issues and difficulties in client server framework that should be considered before being executed in associations, for example, organizations, schools and doctor's facilities [6].

\section{A. Heterogeneity:}

It might exists because of many reasons like distinction in Operating Systems (albeit much work has as of now been done here to beat heterogeneity), diverse in the Bit-Representation, distinctive utilization of Protocols for foundation of association and so forth [7].

B. Expertise

A system administrator is required, which is very exorbitant, to deal with particular specialized errands, for example, the set up of server upkeep together with the treatment of specialized issues [8].

C. Users

No use can be seen if the count of individuals utilizing the workstation is not large on the grounds that a CS system is tedious installation task [9].

D. Server Cost

The necessity of an elite, powerful and solid server doesn't come exceptionally modest. Similarly for operating system used to bolster arranged condition i.e. more costly as compared to individual sorts [10].

E. Server or Network Failure

Procedure of demand of a client is impossible in the earth where the server has an issue or the system association falls flat.

F. Communication Infrastructure Support

The distinctive correspondence interface necessities of various equipment and software stages for clients and servers should be overcome by finding any accessible coordinated administration apparatuses [11].

G. Application Management

The application running for operating system on every clients and servers must be the correct one and accessible by every one such that one of these programs are kept refreshed [12].

\section{PROPOSED SOLUTION}

To accomplish the objective of semantic straightforwardness, the usage of a RPC instrument depends on the idea of stubs, which give an impeccably typical (nearby) methodology call reflection by disguising from applications' interface to basic RPC system. In this way the execution of RPC system includes the accompanying five components of program:
A. The client
B. The client stub
C. The RPC runtime
D. The server stub
E. The server
F. The RPC messages

The client stub, the client, and one occurrence of RPC runtime executes on client system, whereas server stub, server and RPC runtime executes on the server machine shown in fig2.

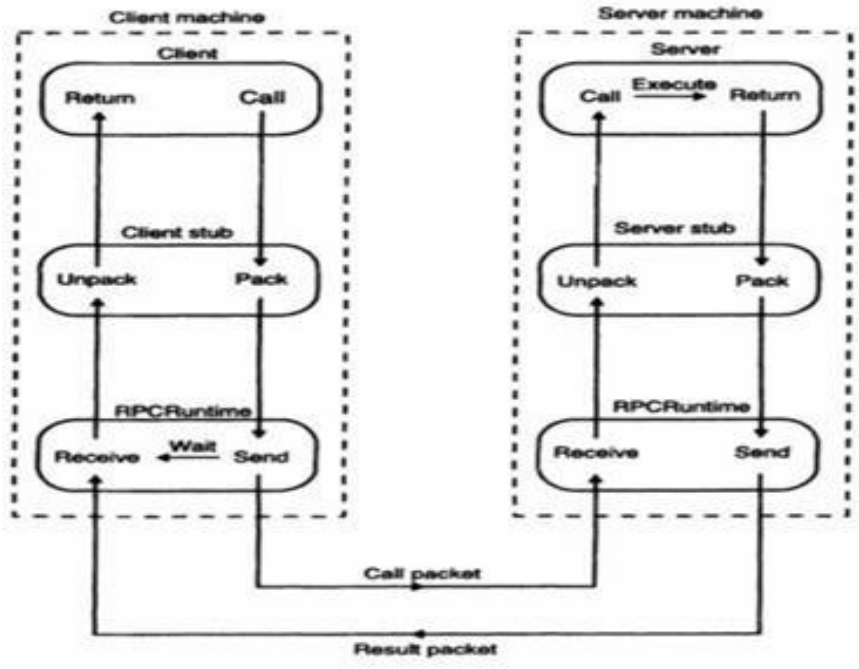

Fig2: RPC mechanism Implementation

\section{A. The client}

It is a client procedure which will start a remote technique call. In order to make a server methodology call, client will make a superbly ordinary nearby call which summons: a comparing technique in client stub. Also, the client will pass his bit representation information. 
B. The client Stub

The Client Stub is in charge of completing the accompanying two assignments:

- On arrival of a request from a client, it will specify the objective technique, the data representation information, the argument is passed in message and afterward the nearby $\mathrm{RPC}$ runtime is asked to send it to the server stub.

- On arrival of the packet, it will unload the outcome and will pass it to the client

\section{The RPC Runtime}

The RPC runtime will handle messages transmission over system amongst server and client system. It will be in charge of retransmissions, affirmations, bundle directing and encryption. RPC runtime on client system and will get the call for messages from client stub and further, will send it to server machine. This additionally will get the messages having the execution result from server system and will pass it to client stub. RPC runtime on server machine will get the messages having the consequence of execution of strategy from server stub and it will be sent to client machine. This will likewise get the message call from client machine and shall then be passed to server stub.

D. The server stub

Occupation of server stub is fundamentally the same as that of client stub. This will play out the accompanying 2 undertakings:

- Reception of call for messages from the nearby RPC runtime, server stub will unload it, also giving the data representation information obtained from the client to the server and will make a superbly ordinary call to summon the proper method in server.

- Reception of the result of method execution from server, server stub will pack the outcome in a message and after that I will ask the neighborhood $\mathrm{RPC}$ runtime to send it to the client stub.
E. The server

On accepting of call demand from the server stub, the server will execute the proper method, it will match the data bit information with its own data representation format, if same it will pass the result in the existing bit representation format, else it will change the result into the client's data bit representation format and then, the result of methodology execution is returned to server stub.

F. The RPC messages

The method of interface between server and client is when client requests will be executed as a remote system by the server and the server will give back the execution result of the related procedure to client. Two sorts of messages required in the usage of RPC systems are takes after:

1) The client sends Call messages to the server for asking for execution of a specific remote strategy.

2) The server sends Reply messages to the client for giving back the consequence of remote technique execution.

G. Messages of Call

Call message is utilized to demand implementation of a specific remote system; 2 essential segments important in call message are as follows:

- Distinguishing proof data of the remote strategy to be executed.

- The contentions vital for the execution of strategy.

- A message distinguishing proof field that comprises of an arrangement number. The field is helpful in 2 routes for recognizing copy messages and lost messages if there should arise an occurrence of system disappointments and for appropriately coordinating answer messages to remarkable call messages, particularly in those situations where the answers of a few exceptional get messages touch base out of request. 
- A message sort field i.e. utilized to recognize calls messages from answer messages. In RPC system, for all call messages, the field might be turned to 0 and to 1 for all answer messages.

- It will also have an additional field of data bit representation which will carry the encoded bits of the corresponding data bit representation, given as:

$$
\begin{aligned}
& \text { 1's complement: } 000 \\
& \text { 2's complement: } 001 \\
& \text { 8's complement: } 010 \\
& \text { 9's complement: } 011 \\
& \text { 15's complement: } 100 \\
& \text { 16's complement: } 101
\end{aligned}
$$

A client recognizable proof field that might be utilized for two reasons: to permit the RPC server to distinguish the client to whom the answer messages should be sent back and to permit server to check for the client confirmation procedure to execute the concerned strategy.

\section{H. Reply messages}

As when the RPC server gets a call messages from a client, it could be confronted with one of the accompanying conditions:-

- The server finds that the call messages are not coherent to it. This may this may happen when a call message disregards the RPC convention

- The server recognizes by examining the client's identifier field that the client is not approved to utilize the administration.

- The server handle that the remote program, rendition, or method number indicated in the remote technique identifier field of the call message is not accessible with it shown in fig3.

- If this stage is achieved, an endeavor will be made to execute the remote method indicated in the call message.

- An exemption condition, (for example, division by zero) happens while executing the predefined remote system.
- The indicated remote method is executed effectively.

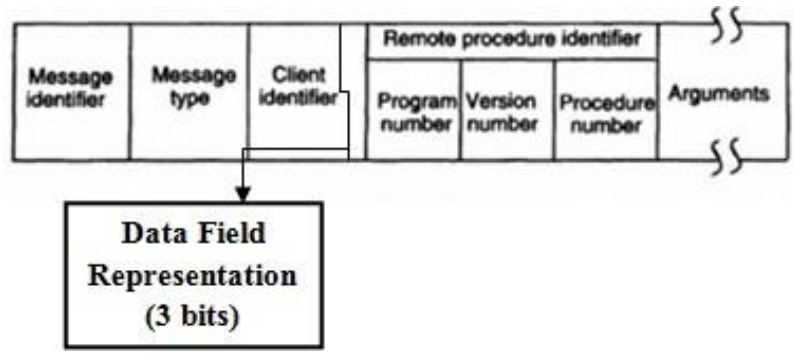

Fig3: Modified RPC Call Message Format.

This field might be added to the call message organize that should give back the bit portrayal of the client's system so that the uniqueness of the information can be kept up and at the Server end the transformation of the Bit-system can be given according to the coveted Bit-Representation of the Client System that will settle the Heterogeneity issue in view of various piece portrayal in the Client Server Systems shown in fig4.

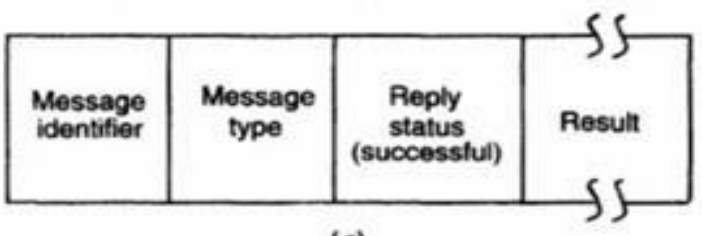

(a)

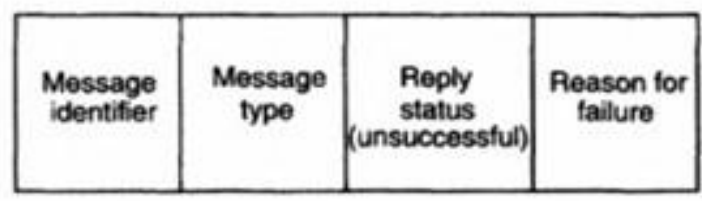

(b)

Fig4. RPC reply message format (a) successful reply message (b) unsuccessful reply message

In this manner, the configuration of an effective answer message and an unsuccessful answer message is somewhat unique. The message sort field is legitimately set to demonstrate that it is an answer message for an effective answer; the answer status field is regularly set to zero and is trailed by the field containing the result of method execution. 


\section{CONCLUSIONS}

This paper has addressed a solution to current problem of Heterogeneity in Client-Server computing based on RPC. In particular we have focused on the potential of RPC to solve the problem. Also, we have expressed a vision of a future Client-Server environment on the Client side, on the Server and between them.

\section{FUTURE SCOPE AND DIRECTIONS}

Client Server model has turned out to be more essential in circulated figuring, deserting the centralized computer innovation regarding its focal points and admiration. Client Server model, all together with its design, has a great deal of opportunities for future development. Further advancements and research in this model have been directed to enhance and deliver good answers for clients. This is imperative for problems and difficulties in the framework to be routed to guarantee that usage of CS framework in accordance with the achievement.

\section{REFERENCES}

[1] Zhang, H. (2013). Architecture of Network and ClientServer model. arXiv preprint arXiv:1307.6665.

[2] Dewire, D. T. (1993). Client/server computing. McGraw-Hill, Singapore

[3] Kratky, S., \&Reichenberger, C. (2013). Client/Server Development based on the Apple Event Object Model. Atlanta

[4] Davis, P.T.(Editor) (1996). Securing Client/Server Computer Networks. McGraw-Hill, USA

[5] Kratky, S., \& Reichenberger, C. (2013). Client/Server Development based on the Apple Event Object Model. Atlanta.

[6] Fong, J., \& Hui, R. (1999). Application of middleware in the three tier client/server database design methodology. Journal of the Brazilian Computer Society, 6(1), 50-64.

[7] Client-Server Model Haroon Shakirat Oluwatosin School of Computing Universiti Utara Malaysia Kedah, Malaysia IOSR Journal of Computer Engineering (IOSR-JCE) e-ISSN: 2278-0661, p- ISSN: 22788727Volume 16, Issue 1, Ver. IX (Feb. 2014), PP 67-71

[8] A. Boussad, A. Said, and L. Jean-Jacques, "Client-server networked automation systems reactivity: deterministic and probabilistic analysis," IEEE Transactions on Automation Science and Engineering, vol. 8, no.3, 2011.

[9] Outtagarts, A. (2009). Mobile agent-based applications: A survey. IJCSNS International Journal of Computer Science and Network Security, 9(11), 331-339.
[10] ] P. E. Renaud, Introduction To Client/Server Systems: A Practical Guide For Systems Professionals, Wiley \& Sons, 1993.

[11] B. D. Imed, "Client-server network architecture for safe pilgrim journey in the kingdom of Saudi Arabia," 2010, IEEE Intelligent Vehicles Symposium, pp. 1043-1048.

[12] ] L. Chih-Cheng, L. Chang-Wu, and C. YI-An, "Integrating security considerations in Client Server architectures of health information systems development," 2011, 5th International Conference on Innovative Mobile and Internet services in Ubiquitous Computing, pp. 527-531. 